\title{
Prise en charge prothétique des fentes orofaciales ò la phase néonatale
}

\section{Prosthetic care of oral and facial clefts at the neonatal period}

\section{MOTS-CLEFS :}

- Obturateurs palatins, orthèses, fentes orofaciales

KEYWORDS:

- Palatal obturators, orthosis,

Oral facial clefts

$\operatorname{AOS} n^{\circ} 284-2017$

\section{Résumé}

Les fentes orofaciales constituent l'un des défauts congénitaux les plus fréquents de nos jours. Leur diagnostic peut se faire dès l'étape prénatale vu le progrès que connaissent les moyens d'investigations radiologiques. La réussite de leur traitement passe par une prise en charge, dans le cadre d'une équipe multidisciplinaire, incluant le chirurgien, l'orthodontiste, le spécialiste en prothèse maxillo-faciale et l'orthophoniste, selon une chronologie d'intervention de chacun, bien définie. À travers ce travail on va essayer de décrire la place de la réhabilitation prothétique dans cet arsenal thérapeutique, les différents appareillages ainsi que la chronologie de leur utilisation en fonction de l'étendue et du siège du défaut facial.
Abstract

Oral facial clefts are one of the most common congenital defects nowadarys. Diagnosis can be made from the prenatal stage to progress experienced by means of radiological investigations. Successful treatment requires a supported, as part of a multidisciplinary team, including the surgeon, orthodontist, specialist in maxillofacial prosthesis and the speech therapist, according to each intervention chronology well defined. Through this work we will try to describe the role of prosthetic rehabilitation in this treatment the various equipment and the timing of their use depending on the extent and the seat of the facial defect.

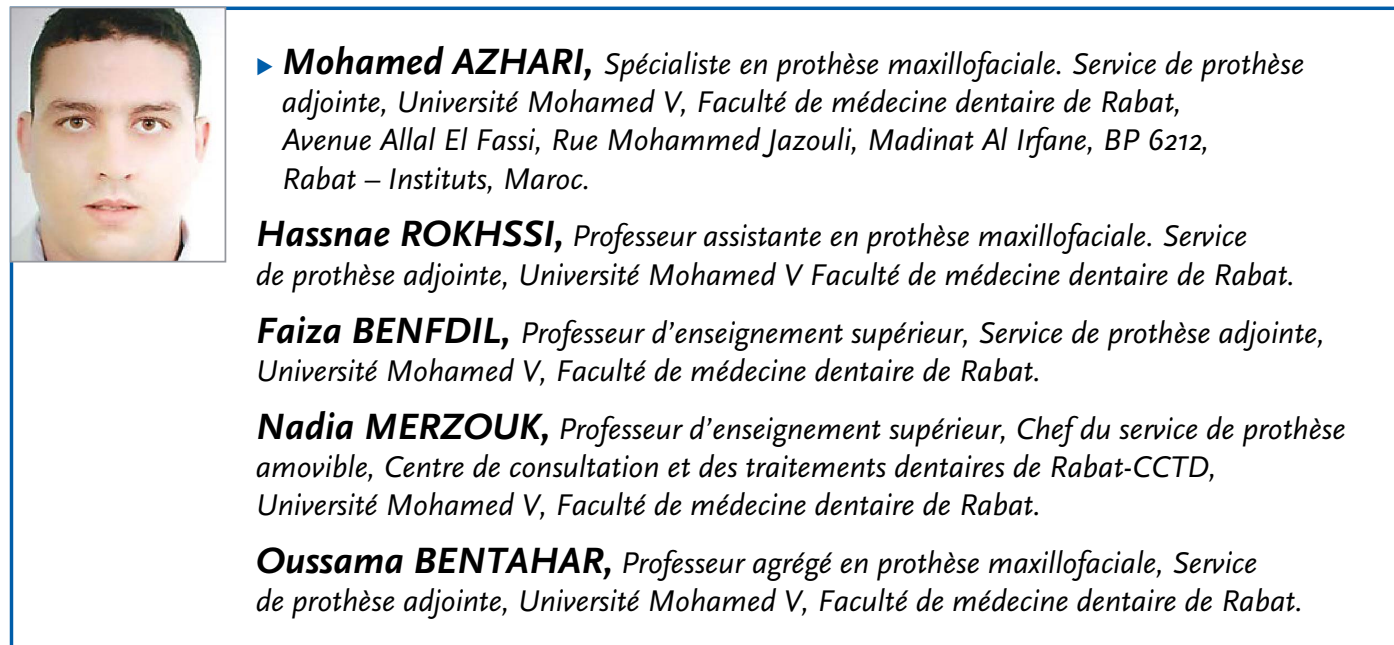




\section{INTRODUCTION}

Les fentes orofaciales, pouvant être à localisation labiale, alvéolaire, palatine, vélaire ou intéressant une ou plusieurs de ces zones. Elles constituent l'un des défauts congénitaux les plus fréquents de nos jours. Leur diagnostic peut se faire dès l'étape prénatale vu le progrès que connaissent les moyens d'investigations radiologiques. Le traitement de ces fentes est principalement chirurgical, mais la place de la thérapeutique prothétique et orthopédique est d'une grande importance dans le succès d'un tel arsenal thérapeutique. Ces plaques peuvent être utilisées uniquement pour obturer le défaut congénital, on parle alors de plaques palatines passives, comme on peut leur associer une action orthopédique, c'est le cas des plaques actives.

La prise en charge prothétique de ces défauts congénitaux peut continuer même à l'âge adulte, c'est le cas des fentes qui subsistent après l'acte chirurgical, on parle des fentes séquellaires ou résiduelles. Ces fentes intéressent généralement le voile du palais et à moindre degré le palais dur. Selon leur étendue et leur siège, ces défauts peuvent nécessiter des obturateurs spécifiques à chaque situation clinique. $S^{\prime}$ il s'agit d'une fente vélaire résiduelle, la réhabilitation va dépendre de l'état morphologique et fonctionnel du moignon vélaire. En effet, dans les cas de voiles divisés ou courts mais gardant une tonicité relativement normale, l'obturateur de SUERSEN pour ceux divisés ou celui de Schiltsky pour ceux courts seront indiqués; par contre si le voile est inerte ou même absent, on opte respectivement soit pour l'obturateur de MAZAHERI ou soit pour celui de Fröschel et Schalit.

Cet article est consacré à la phase néonatale, vu l'importance que présente la réhabilitation prothétique dans cette étape de la vie permettant d'une part le rétablissement des fonctions orales souvent altérées et d'autre part l'amélioration du terrain pour un éventuel acte chirurgical et à travers ceci une intégration optimale des parents dans la prise en charge de leur bébé.

\section{DÉFINITION}

Les fentes orofaciales sont des défauts congénitaux, résultant d'une absence de fusion entre les bourgeons faciaux lors de la 4 e semaine de la vie intra-utérine et se manifestent par une absence ou insuffisance de fusion de la lèvre supérieure, et/ou du rebord alvéolaire du maxillaire et/ou du palais osseux et/ou du voile du palais. [1]

\section{ÉTIOPATHOGÉNIE}

La plupart des auteurs s'accordent sur le fait que les fentes orofaciales ont une origine multifactorielle, avec une interaction complexe entre des facteurs génétiques à pénétrance et seuils d'expression variables, modulés par des facteurs environnementaux. Ce caractère multifactoriel rend par ailleurs le conseil génétique aux parents difficile et empirique.

I L'hérédité joue certainement un rôle dans la transmission des fentes faciales, ainsi tous collectifs confondus, des grandes séries ont montré qu'un tiers des cas ont une histoire familiale positive pour ce type de malformation. Toutefois un mode précis de transmission héréditaire n'est pas applicable. Dans certaines grandes familles, une transmission mendélienne a été évoquée. [2]

I Plusieurs gènes défectueux ont été étudiés et mis en relation avec les fentes faciales (TGFa, TGFb, MSX1, RARa...) avec des associations alléliques et des pénétrances variables. [3]

1 Certains facteurs environnementaux peuvent intervenir ainsi, en expérimentation animale, des fentes faciales ont été produites par des déficiences en vitamine $\mathrm{A}$, riboflavine, acide folique ou un excès de cortisone. Chez l'homme, plusieurs tératogènes ont été mis en relation avec l'apparition de fentes faciales : tabac, alcool, rétinoïdes, aminoptérine, diphénylhydantoïnes, triméthadione, thalidomide. [4]

\section{CLASSIFICATION}

Plusieurs classifications des fentes orofaciales ont été proposées dans la littérature, celle de BEUMER (Fig. 1) nous semble plus simple et utile en termes de la thérapeutique prothétique. [5]

\section{OBSERVATIONS CLINIQUES}

\section{Premier cas clinique: \\ FLAP unilatérale complète traitée avec une plaque passive}

\section{Examen clinique et pronostic}

Il s'agit d'un nouveau-né présentant une FLAP unilatérale complète (Fig. 2), l'examen général a montré que le patient ne présente aucune pathologie générale et aucun défaut malformatif associé, l'examen local et locorégional permet de déterminer l'étendue de la fente ainsi que les éléments anatomiques favorables et défavorables pour une éventuelle réhabilitation prothétique, présentés selon un tableau récapitulatif (Tableau 1) permettant de donner un score pour le 
Prise en charge prothétique des fentes orofaciales à la phase néonatale Numéro spécial Pathologie - 1

A. Absence de fente

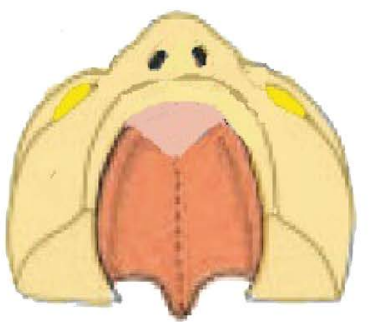

D. Dévision du palais et du prémaxillaire

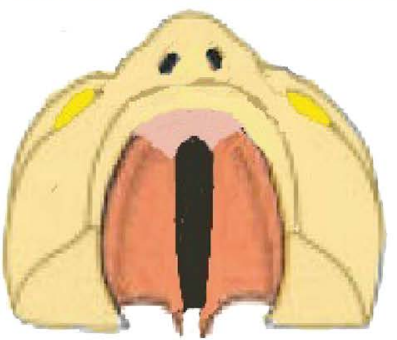

G. Dévision labiale et prémaxillaire bilatérale assiciée à une dévision palatine unilatérale

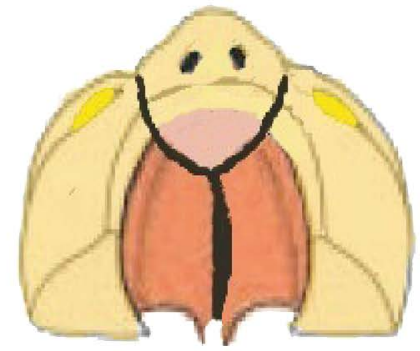

B. Dévision de la luette seulement

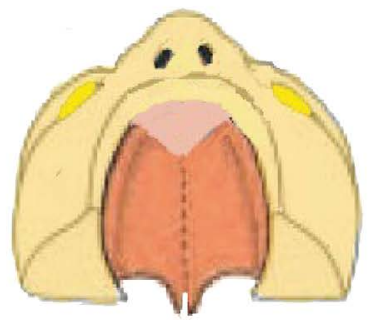

E. Dévision unilatérale du prémaxillaire et de la lèvre

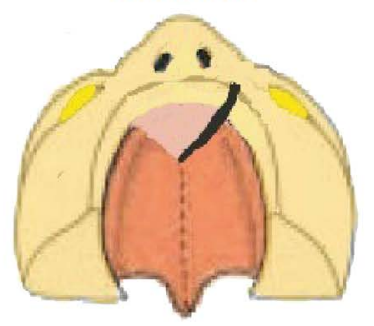

C. Dévision du palais mou et palais dur

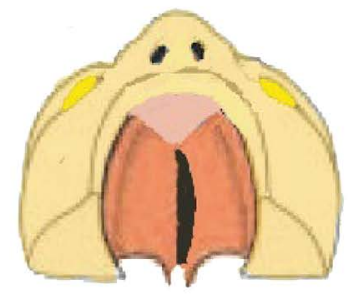

F. Dévision bilatérale du prémaxillaire et de la lèvre

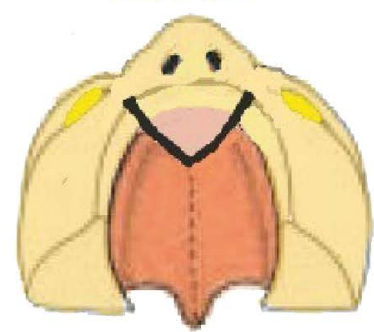

H. Dévision bilatérale de la lèvre,
du prémaxillaire et du palais

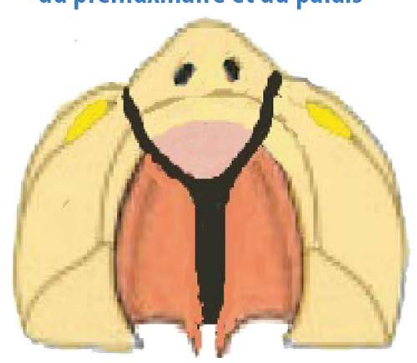

$\triangle$ Fig. 1 : Classification des fentes orofaciales selon Beumer J et al.

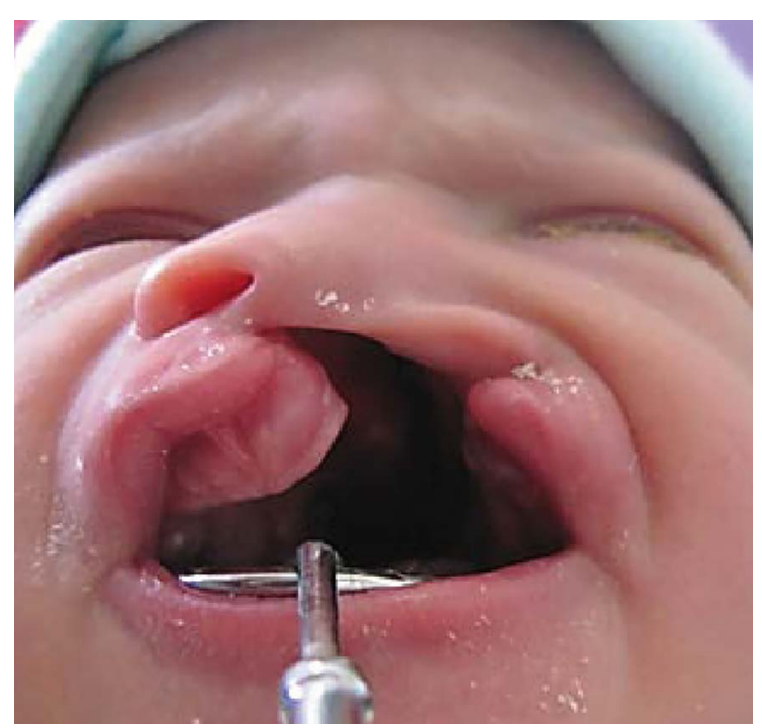

$\triangle$ Fig. 2 : Fente labio-alvéolo-palatine unilatérale gauche.

\begin{tabular}{|c|c|c|}
\hline Facteurs anatomiques & Favorables & Défavorables \\
\hline $\begin{array}{l}\text { Le volume osseux } \\
\text { (tubérosités-crêtes) }\end{array}$ & + & \\
\hline $\begin{array}{l}\text { L'état des fibromuqueuses } \\
\text { (épaisseur, texture, } \\
\text { adhérence) }\end{array}$ & + & \\
\hline $\begin{array}{l}\text { La denture } \\
\text { (édenté ou denté) }\end{array}$ & & + \\
\hline $\begin{array}{l}\text { Le flux salivaire } \\
\text { (fluide - visqueux) }\end{array}$ & + & \\
\hline $\begin{array}{l}\text { La langue (situation - } \\
\text { volume - tonicité) }\end{array}$ & + & \\
\hline $\begin{array}{l}\text { La sangle labio- } \\
\text { jugale(situation - tonicité) }\end{array}$ & & + \\
\hline $\begin{array}{l}\text { Le voile du plais } \\
\text { (le reste - tonicité) }\end{array}$ & + & \\
\hline $\begin{array}{l}\text { L'étendue de la fente } \\
\text { (complète - partielle) }\end{array}$ & & + \\
\hline $\begin{array}{l}\text { Score du pronostic } \\
\text { prothétique }\end{array}$ & 5 & 3 \\
\hline
\end{tabular}

$\triangle$ Tableau 1 : Établissement de score à travers les indices biologiques de la surface d'appui prothétique. 
pronostic de notre conception prothétique ; ainsi pour cette situation clinque, le pronostic est favorable.

\section{Étapes prothétiques}

L'empreinte chez le nouveau-né semble être un acte difficile, nécessitant l'habilité et la patience du praticien. Le recours à l'anesthésie générale est le plus souvent écarté, mais l'oxygénothérapie est par contre conseillée ; cet acte est préférablement effectué en l'absence des parents pour éviter tout traumatisme psychologique et donc perturbation du geste opératoire. Il est réalisé à quatre mains ce qui veut dire que l'aide est obligatoire.

Selon les équipes, la position du nourrisson varie. Au CCTD de Rabat nous avons opté depuis longtemps pour une position assise avec la tête bien soutenue dans le bras de l'aide et dans la main gauche de l'opérateur. Cette position a le double avantage de faciliter l'acte de l'empreinte et de contrôler à tout moment la liberté des voies aériennes (Fig. 3). Dans le cas de la séquence de Pierre Robin la position de décubitus ventral est souhaitable vue les difficultés respiratoires qui peuvent être aggravées par la prise de l'empreinte. Il faut veiller également à ce que l'enfant fasse des mouvements de succion pendant l'empreinte car cela permet d'enregistrer aussi physiologiquement que possible les structures anatomiques para-prothétiques. Les pleurs du bébé sont un signe important du bon déroulement de l'empreinte parce que ça indique que les voies aériennes ne sont pas obstruées par la langue ou le matériau à empreinte.

L'empreinte est réalisée, si possible, à l'aide d'un porte-empreinte de série pédiatrique; mais le plus souvent l'absence de l'adaptation de ces porte-empreintes, nous pousse toujours à confectionner des portes-empreintes individuels (PEI) soit sur des modèles de travail issus des patients pris en charge auparavant soit en utilisant des supports façonnés en

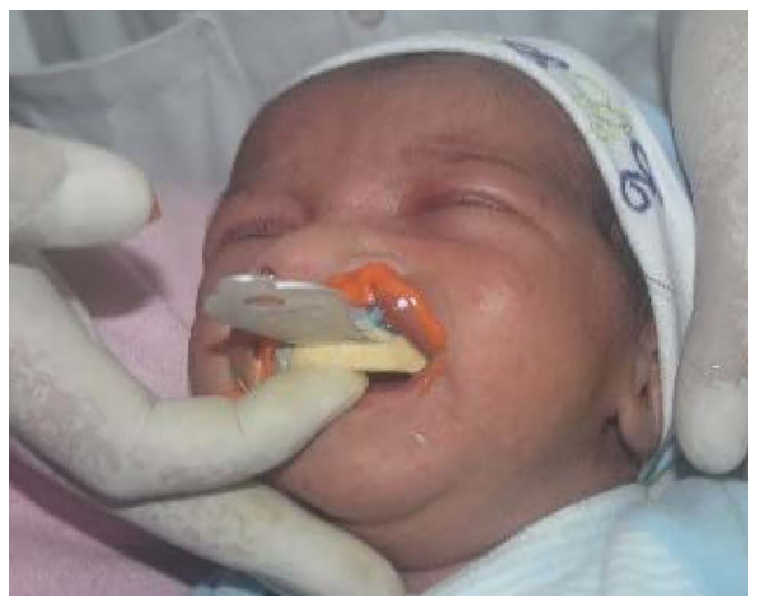

$\triangle$ Fig. 3 : Technique d'empreinte selon notre expérience : main gauche du praticien maintient la tête du nourrisson au niveau de la région occipitale, sa main droite s'occupe de l'empreinte. l'occurrence un doigtier de gant, un feuillet de cire adapté au préalable sur la surface d'appui ou même des matériaux théâtraux « la tulle » [5], servant donc pour véhiculer le matériau à empreinte sans risque de fusion dans les voies aérodigestives supérieures. Cependant la technique la plus précise et la plus recommandée est celle qui est adoptée par notre équipe en utilisant des porte-empreintes de différentes dimensions façonnés sur un jeu des anciens modèles en plâtre (Fig. 4).

Selon notre expérience, la technique d'empreinte est faite en deux temps. Les matériaux à empreinte les plus utilisés sont les élastomères type silicone, du fait de leur stabilité volumétrique, leur goût agréable, leur présentation sous forme de plusieurs viscosités, et leur grande précision d'enregistrement. Les hydrocolloïdes irréversibles doivent être évités afin de prévenir tout risque d'inhalation du matériau ou rétention de ses débris dans les zones de contre-dépouilles nasales et para-nasales. Le PEI est ajusté en bouche, en éliminant toute zone de surextension ou de compression, puis garni d'adhésif avant la prise d'empreinte. En premier temps l'empreinte est réalisée à l'aide d'un silicone de haute viscosité (Fig. 5), rebasée dans un

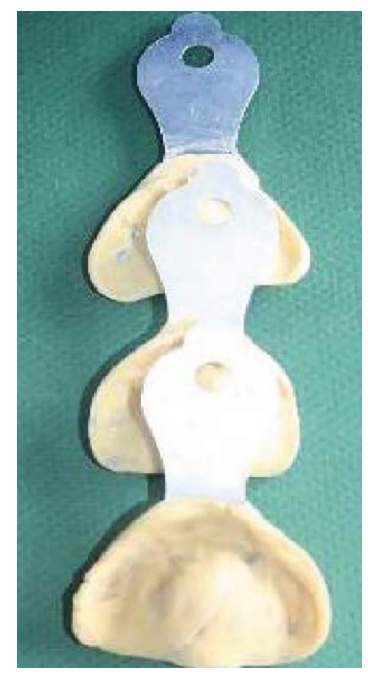

4 Fig. 4: Série de porteempreintes individuels selon plusieurs dimensions façonnés sur des modèles de patients pris en charge auparavant.

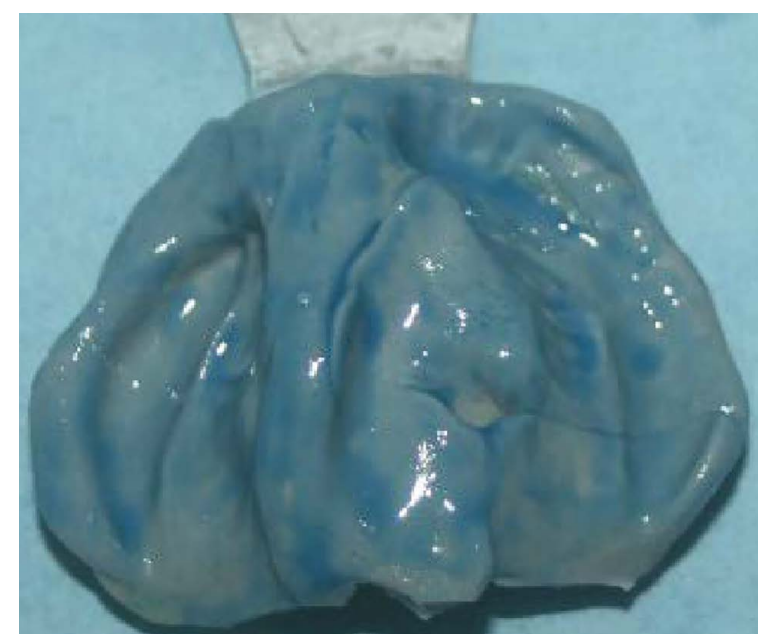

$\triangle$ Fig. 5 : Premier temps d'empreinte : empreinte globale à l'aide d'un élastomère haute viscosité. 
second temps avec un matériau silicone de basse viscosité (Fig. 6).

L'empreinte est traitée puis coulée à l'aide de plâtre dur ou extradur, ce qui va permettre d'avoir un modèle de travail sur lequel on va pouvoir confectionner une plaque palatine (Fig. 7).

Le modèle de travail est d'abord modifié en comblant les éventuelles contre-dépouilles surtout au niveau de la zone de la fente en l'occurrence à l'aide de cire rose (Fig. 8), puis isolé à l'aide de vernis de laboratoire (fig. 9), avant de commencer à apporter de manière alternative la résine poudre et liquide (technique de saupoudrage) en essayant de couvrir au maximum toute la surface d'appui (Fig. 10). La plaque est polymérisée, dégrossie, polie et brillantée (Fig. 11).

La séance de l'insertion en bouche, doit être prise avec beaucoup de rigueur par le praticien qui doit en fait, pouvoir répondre aux attentes des parents. C'est-à-dire le rétablissement des fonctions orales notamment la succion et la déglutition. Pour ce faire un ajustement de la plaque doit être réalisé au préalable par des éventuelles retouches et contrôles en tirant profit de la transparence de la plaque pour éliminer toute zone de compression et de surextension.

Le suivi consiste en un contrôle et une stimulation périodique et continue de l'avancé des brèches de la fente, en réalisant des modifications sur la plaque par soustraction ou par adjonction de la résine. En effet, le résultat attendu par cette plaque n'est pas proprement dit la fermeture de la fente mais surtout la reconstitution des fonctions orales altérées et l'adaptation des muscles périphériques avec ces fonctions, chose qui va faciliter par la suite l'acte chirurgical.

\section{Deuxième cas clinique : fente palatine syndromique (séquence de Pierre Robin)}

\section{Examen clinique}

Il s'agit d'un nouveau-né âgé de 2 mois présentant une fente vélopalatine limitée au plais dur et au voile du palais (Fig. 12, 13) ; l'examen clinique général permet de confirmer que ce défaut rentre dans le cadre de la séquence de Pierre Robin qui se manifeste en outre par une rétrognathie mandibulaire et une glossoptose. Le motif de consultation était surtout la réhabilitation fonctionnelle (la succion et la respiration perturbées). Une plaque palatine passive a été indiquée permettant de remédier aux problèmes fonctionnels et d'inciter les fragments à se rapprocher au maximum par des retouches sélectives avant l'instauration de l'acte chirurgical.

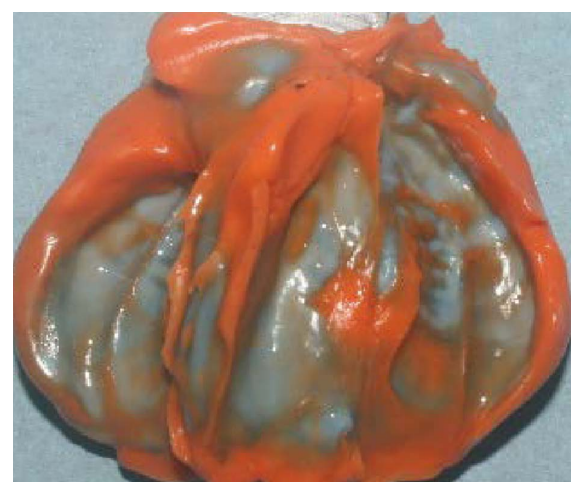

$\triangle$ Fig. 6 : Deuxième temps d'empreinte : empreinte de rebasage à l'aide d'un élastomère de basse viscosité.

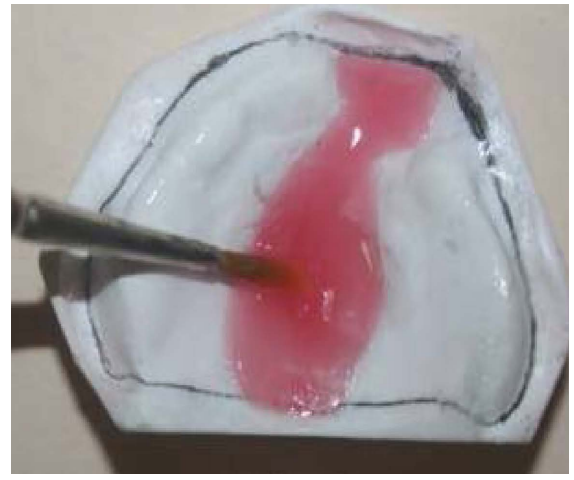

$\triangle$ Fig. 9 : Isolation de la surface d'appui avec un vernis de laboratoire.

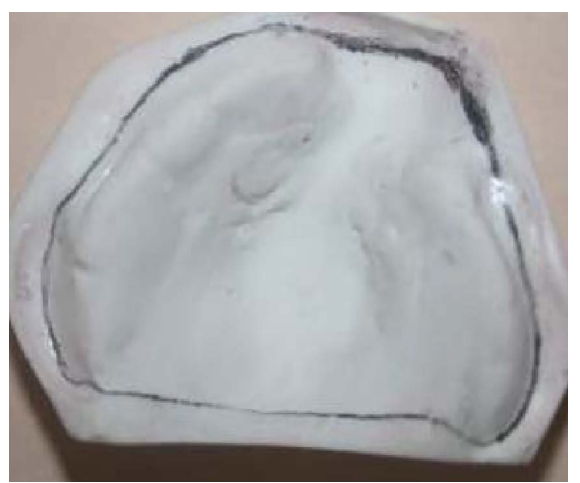

$\triangle$ Fig. 7 : Modèle de travail issu de l'empreinte et tracé des limites de la plaque palatine.

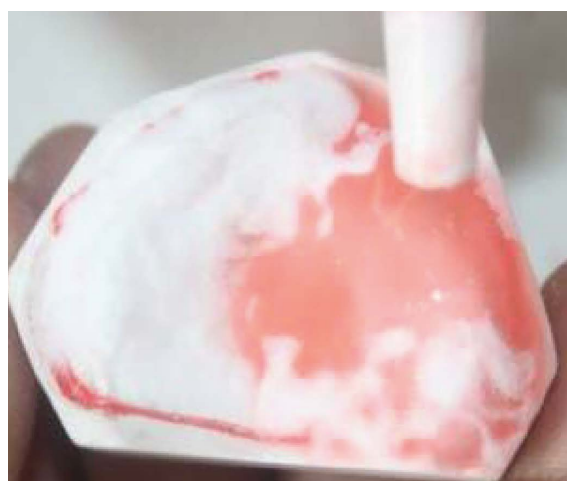

$\triangle$ Fig. 10: Technique de saupoudrage avec alternance poudre liquide de la résine autopolymérisable transparente.

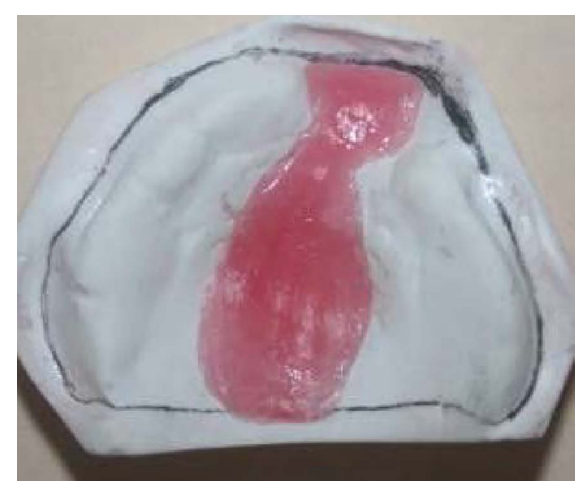

$\triangle$ Fig. 8 : Comblement de la zone de la fente à l'aide de cire.

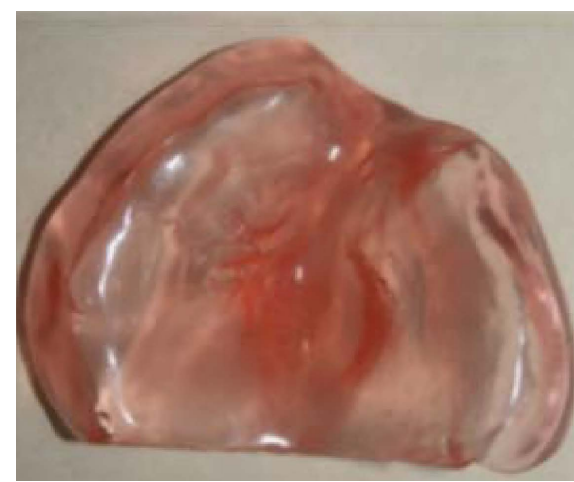

$\triangle$ Fig. 11 : plaque palatine après dégrossissage, finition, polissage et brillantage (vue d'extrados et intrados). 


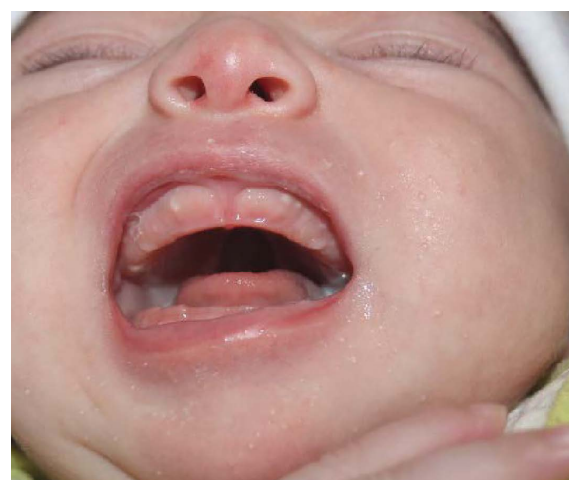

$\triangleleft$ Fig. 12 : Syndrome de Pierre-Robin : fente palatine, glossoptose et rétrognathie mandibulaire.

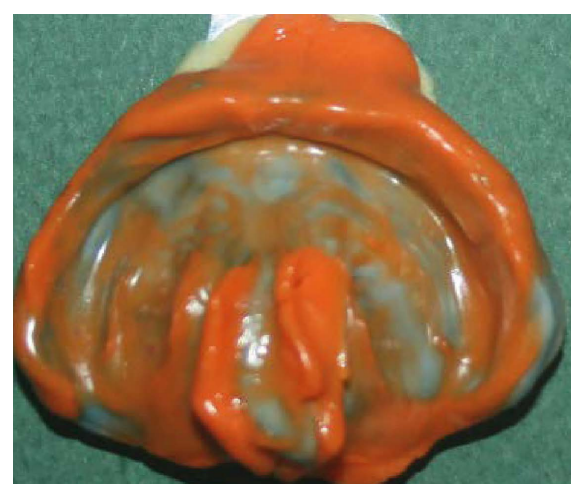

$\triangle$ Fig. 15: Empreinte de précision à l'aide d'élastomère basse viscosité. Il faut accélérer autant que possible la prise par adjonction d'une grande quantité d'activateur pour réduire au maximum le temps d'empreinte en bouche.

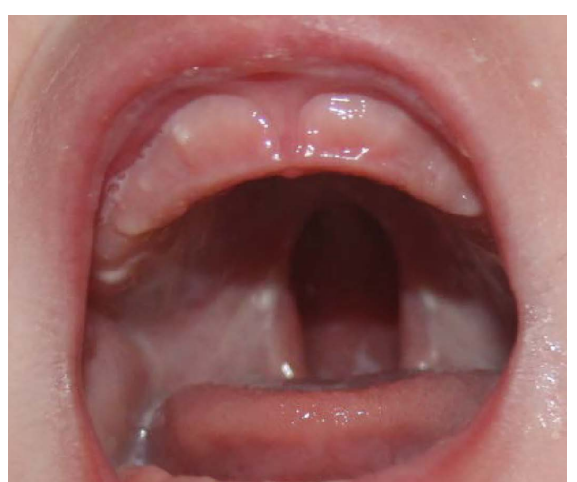

$\triangle$ Fig. 13 : Examen clinique permettant de déceler l'étendue et le siège de la fente palatine.

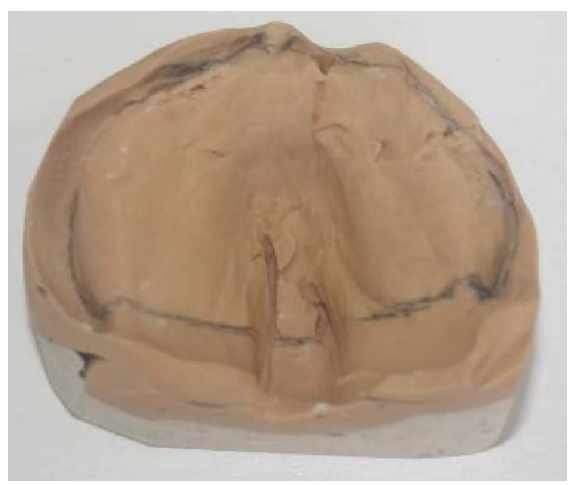

$\triangle$ Fig. 16: Modèle de travail issu de l'empreinte.

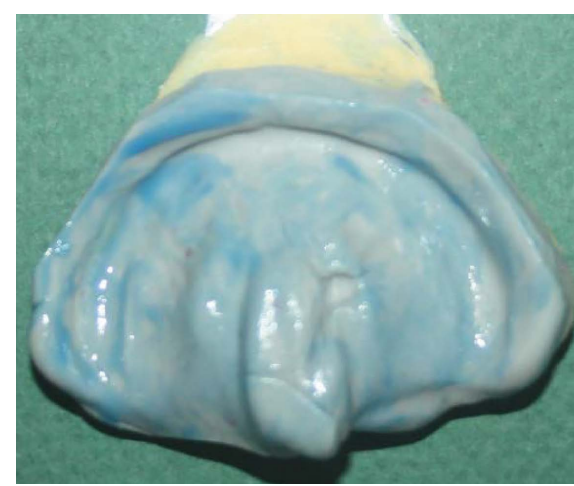

$\triangle$ Fig. 14 : Empreinte globale à l'aide d'un silicone haute viscosité permettant d'enregistrer le siège du défaut palatin.

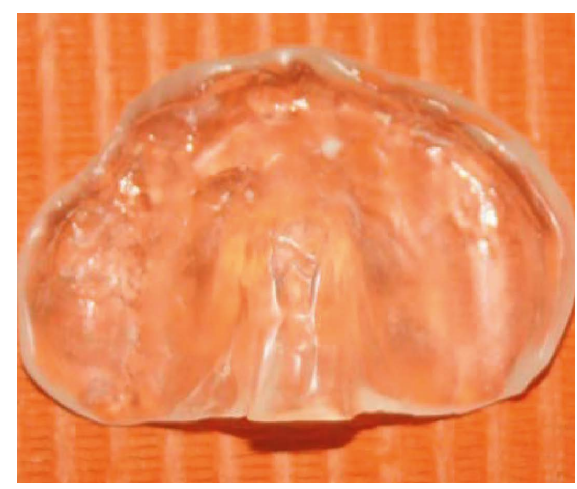

$\triangle$ Fig. 17: Plaque palatine réalisée selon la technique de saupoudrage de la résine polyacrylique autopolymérisable.

\section{Étapes prothétiques}

L'empreinte est réalisée à l'aide d'un porte-empreinte confectionné sur un modèle d'étude d'un ancien patient, le matériau à empreinte est un silicone de haute viscosité rebasé avec la basse viscosité dans un second temps (Fig. 14, 15).

Sur le modèle de travail (Fig. 16), une plaque en résine polyacrylique transparente est confectionnée selon le protocole déjà décrit (Fig. 17). La pose et le contrôle en bouche obéissent aux mêmes principes précités.

\section{Troisième cas clinique :}

plaque palatine passive associée

à un conformateur orthopédique nasolabial

Il s'agit d'un nourrisson âgé d'un mois qui présente une fente labioalvéolopalatine complète unilatérale avec un effondrement important du cartilage nasal en rapport avec le défaut (Fig. 18). La décision thérapeutique prothétique a été de réaliser une plaque platine associée à un conformateur orthopédique nasolabial. Le premier temps prothétique consiste en la réalisation d'une plaque palatine en suivant les mêmes principes préalablement décrits, avant d'entamer les étapes d'élaboration du conformateur nasolabial proprement dit qui va être détaillé à travers ce cas clinique (Fig. 19). L'empreinte de l'orifice nasal est prise à l'aide d'un silicone lourd en prenant appui sur la plaque bien adaptée en bouche et préalablement enduite au niveau de sa partie antérieure par un adhésif universel (Fig. 20). Après durcissement, le tout est désinséré (Fig. 21) et coulé en plâtre pour obtenir un modèle de travail secondaire modifié sur lequel on va confectionner la seconde partie de l'orthèse (Fig. 22). Au niveau du bord antérieur de la plaque palatine, on réalise une petite rainure pour pouvoir intégrer le plus parfaitement possible le fil métallique. Ce fil qui est choisi de préférence flexible (fil jonc de double longueur et de $0,8 \mathrm{~mm}$ de diamètre) est façonné sur le modèle de manière centrée sans pour autant interférer avec les parois narinaires. Une phase de remplissage est menée en étalant la résine polyacrylique autopolymérisable selon la technique d'alternation poudre et liquide sur le modèle de travail dans la région de l'orifice nasal jusqu'à ce qu'on obtienne une sorte de bouton qu'on va appeler le bouton nasal. Le grattage consiste en 


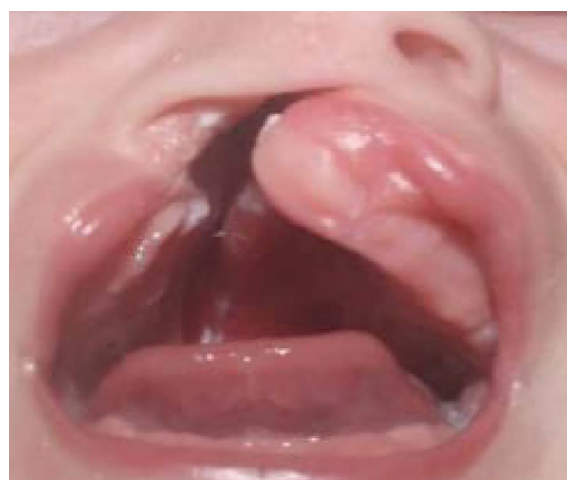

$\triangle$ Fig. 18: Examen exobuccal : fente unilatérale droite avec effondrement du cartilage nasal du même côté.

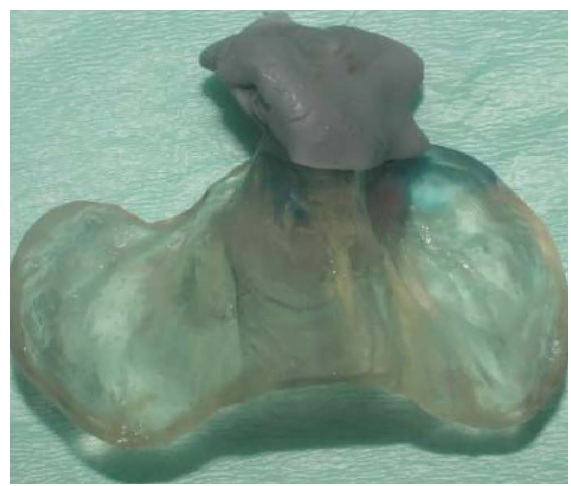

$\triangle$ Fig. 21 : Empreinte de l'orifice nasal à l'aide d'un silicone lourd en utilisant comme support la plaque palatine préalablement adaptée en bouche.

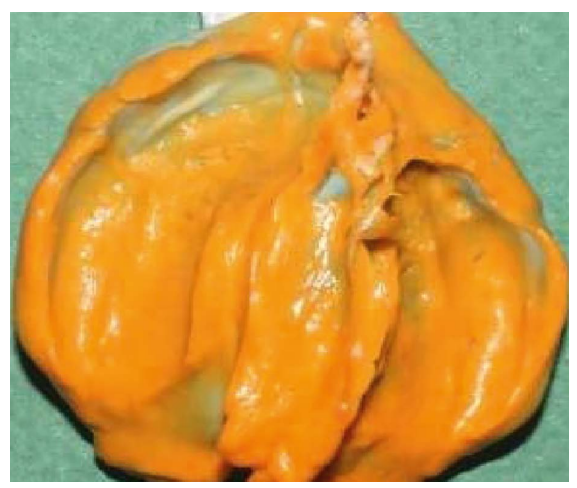

$\triangle$ Fig. 19: Empreinte aux élastomères en deux temps, selon les mêmes principes d'empreintes pour plaque palatine passive préalablement décrits dans les deux cas précédents.

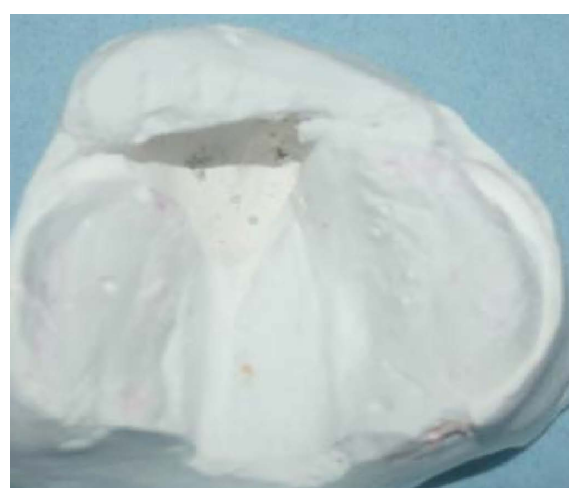

$\triangle$ Fig. 22 : Modèle issu de la coulée de l'intrados de la plaque et de l'empreinte du conformateur nasal.

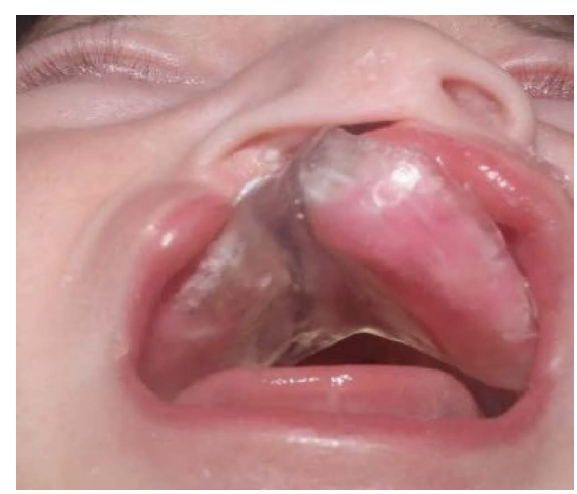

$\triangle$ Fig. 20: Plaque palatine passive issue de l'empreinte. Adaptation en bouche par élimination des zones de compression en utilisant la qualité transparente de la plaque.

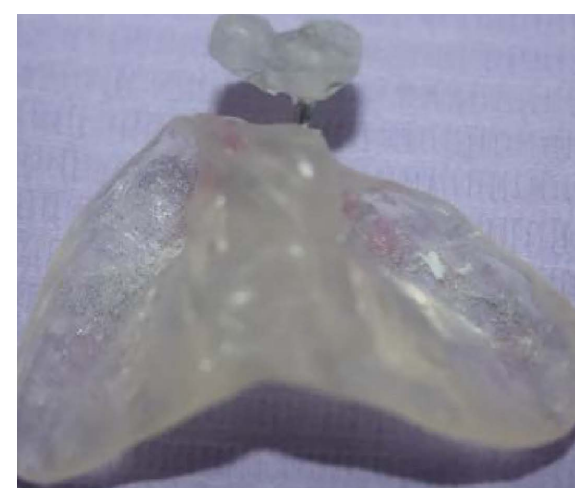

$\triangle$ Fig. 23 : Conformateur naso-labial : le fil métallique de connexion est façonné de manière à ce que son extrémité antérieur soutienne le bouton nasal et son extrémité postérieure soit intégrée dans la masse de la plaque. Le bouton nasal est réalisé par la technique classique de saupoudrage de la résine autopolymérisable. la séparation du bouton de la plaque palatine en ne laissant que le fil métallique qui assurera la liaison entre les deux segments prothétiques (Fig. 23). Après finition et polissage, l'insertion en bouche doit être effectuée en respectant au maximum les éléments anatomiques oraux et nasaux. Le bouton nasal doit pouvoir assurer au maximum le soutien du cartilage nasal sans obturer complètement l'orifice nasal afin de libérer la respiration (Fig. 24). Le suivi consiste en un contrôle continu par activation du fil ou mieux encore par rebasage du bouton pour augmenter le soutien périodiquement toutes les deux semaines jusqu'au temps de la chéiloplastie.

\section{DISCUSSION}

Selon des études épidémiologiques, les fentes labioplatines associées sont les plus fréquemment diagnostiquées (46\%), suivies par les fentes palatines isolées (33\%), puis les fentes labiales (21\%). Les

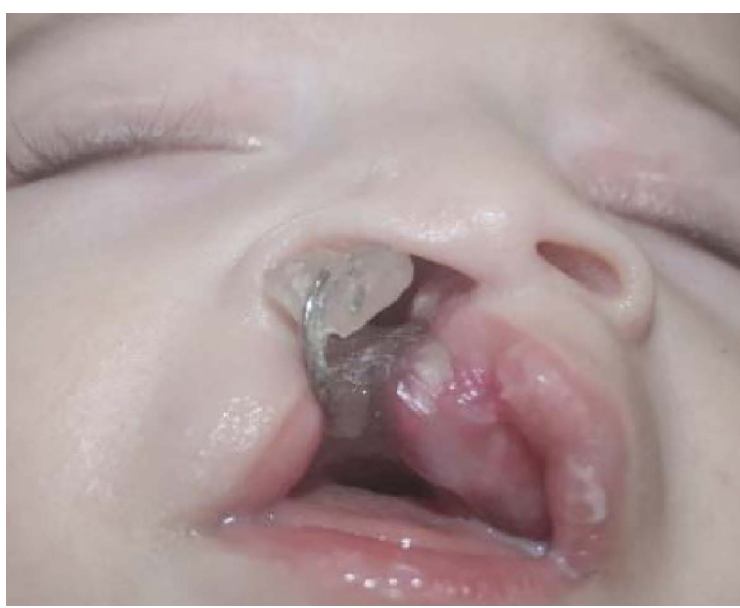

$\triangle$ Fig. 24 : Pose et contrôle. Il faut que le conformateur assure le soutien du cartilage nasal sans pour autant obstruer l'orifice nasal.

fentes unilatérales sont neuf fois plus prédominantes que les fentes bilatérales, et touchent deux fois plus le côté gauche que le côté droit. Les fentes labiopalatines combinées sont dominantes chez le sexe masculin alors que les fentes palatines isolées sont plus fréquentes 
chez le sexe féminin. Dans la population européenne, la fente labiale avec ou sans fente palatine associée survient sur environ 1 sur 1000 naissances vivantes. Ces entités sont deux fois plus fréquentes chez la population asiatique, et environ la moitié chez la population afro-américaine. [7]

Selon les auteurs et le groupe de patients étudiés, de $44 \%$ à $95 \%$ des cas de fentes orofaciales sont accompagnées d'une malformation mineure. Parmi celles-ci, on retrouve des hernies ombilicales, des anomalies des membres (en particulier des doigts et des orteils), des anomalies des oreilles, des malformations cardiaques et un retard mental. [8]

Les fentes orofaciales accompagnent fréquemment des syndromes génétiques, parmi ceux-ci (plus de 340 ont été décrits) : le syndrome de Van DerWoude, le syndrome de Franceschetti-Zwahlen-Klein (ou dysostose mandibulo-faciale), le syndrome de Digeorge et le syndrome ou plutôt la séquence de Pierre Robin qui comprend une tétrade sémiologique obligatoire : une micromandibulie, une glossoptose, une division palatine et un syndrome apnéique du nouveau-né. $[5,9,10]$ L'échographie anténatale permet de diagnostiquer la fente labiale dès le deuxième trimestre de la vie intra-utérine. En revanche, le diagnostic différentiel échographique entre une fente palatine isolée et associée reste encore difficile. Ce diagnostic anténatal a bouleversé le climat de la naissance. En effet ce diagnostic est d'une grande utilité pour la thérapeutique à envisager, car il permet véritablement de mener un plan de traitement précoce, global et de longue durée abordé juste après la naissance. [10]

Selon de nombreuses écoles, les fentes labiales sont corrigées chirurgicalement, généralement en période néonatale (dix premiers jours de vie), et les fentes palatines à l'âge de trois mois. [11] De multiples techniques de correction existent mais la chirurgie à elle seule ne peut pas résoudre le défaut dans son intégralité ainsi que ses complications, d'où l'importance d'y associer une thérapeutique prothétique ou orthopédique en plus d'une rééducation orthophonique.

Dans le but de faire le point sur la place et la chronologie de la thérapeutique prothétique et orthopédique dans l'arsenal thérapeutique des fentes orofaciales, il nous semble plus judicieux de décrire préalablement le calendrier des actes chirurgicaux ; celui-ci est l'objet d'une controverse concernant le principe et la chronologie. En effet la fermeture chirurgicale de ces défauts diffère selon la technique abordée pour chaque école ; les différentes techniques sont schématisées selon le tableau ci-dessous selon la chronologie et le type de fente (Tableau 2). L'indication du type de plaque palatine à utiliser doit se faire toujours en concertation avec les différentes parties de l'équipe multidisciplinaire s'engageant dans le traitement.

La plaque palatine obturatrice appelée aussi selon les auteurs, un obturateur palatin ou un appareil obturateur d'alimentation néonatale est traditionnellement fabriquée à partir d'une résine acrylique transparente mais d'autres matériaux souples peuvent être aussi utilisés. Cet artifice prothétique doit répondre aux objectifs suivants [12] :

réduire les régurgitations nasales,

$\checkmark$ réduire le temps nécessaire à l'alimentation,

$\checkmark$ réduire le risque de blessures des tissus mous en raison de sa texture douce,

permettre de positionner la langue hors de la zone fente dans la position correcte pour permettre la croissance spontanée des lames palatines l'une vers l'autre,

permettre la création d'une pression entre la langue et la plaque palatine favorisant la succion,

$\checkmark$ permettre une alimentation normale et donc une récupération du poids,

I et réduire la frustration des parents en raison des problèmes d'alimentation.

De point de vue terminologique dans le domaine de la réhabilitation prothétique, il faut distinguer les

\section{Calendrier thérapeutique des fentes faciales}

\begin{tabular}{|l|l|}
\hline $0-3$ mois & Calendrier thérapeutique des fentes faciales \\
\hline $3-4$ mois & Chirurgie réparatrice labiale et alvéolaire \\
\hline 7 mois & $\begin{array}{l}\text { Test auditif : si présence de liquide au niveau de l'oreille moyen (signe d'otite) } \rightarrow \text { insertion de l'aérateur } \\
\text { Transtympanique lors de la palatoplastie }\end{array}$ \\
\hline 9 mois & $\begin{array}{l}\text { Absence d'apnée centrale ou obstructive : chirurgie réparatrice du palais } \\
\text { Présence d'apnée centrale ou obstructive : réparation palatine retardée jusqu'au 12 à 18 mois }\end{array}$ \\
\hline $4-12 a n s$ & Pharyngoplastie si nécessaire \\
\hline $12-14$ ans & Greffe osseuse alvéolaire (pour de larges fentes de l'alvéole) \\
\hline $14-16$ ans & Chirurgie esthétique sur les lèvres ou le nez, si nécessaire \\
\hline $16-21$ ans & Chirurgie d'avancée maxillaire si nécessaire \\
\hline
\end{tabular}

Tableau 2 : Chronologie de prise en charge des fentes orofaciales 
prothèses qui sont des appareillages de remplacement et les orthèses qui assurent une fonction orthopédique. Dans ce sens une plaque palatine obturatrice est considérée à la fois comme une prothèse en remplaçant le palais et/ou les dents et une orthèse en permettant un repositionnement de la langue et une action orthopédique sur les fragments de la fente. On distingue deux types de plaques palatines : passives et actives.

\section{Plaques palatines passives}

Les plaques palatines passives sont conçues pour pouvoir normaliser la position de la langue et permettre une déglutition physiologique, une alimentation facile et une croissance harmonieuse et guidée des maxillaires. Elles sont réalisées dès la naissance et portées en continu jusqu'à l'avéoloplastie si l'intervention se déroule en deux temps, ou jusqu'à la palatoplastie si elle est réalisée en un seul temps. [13, 14]

Le suivi régulier est d'une grande importance pour suivre la croissance squelettique et l'éruption des dents. Les modifications sont faites soit par soustraction ou par adjonction de la résine dure ou molle (Fig. 25).

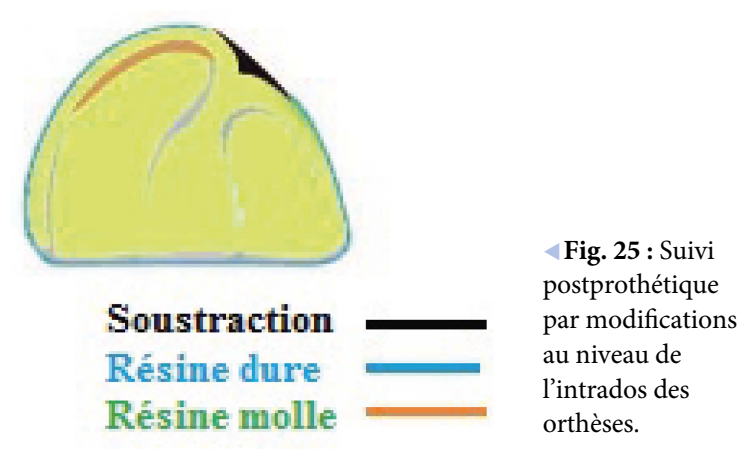

\section{Plaques palatines actives}

Actuellement, le concept de plaques orthopédiques nasolabiales est le plus adopté. $[15,16]$ Ce sont des appareillages popularisés par Grayson et qui visent en plus de l'action de l'obturation de la fente palatine un modelage des tissus mous et des cartilages nasaux par un conformateur solidaire à la plaque palatine. Ce sont des artifices indiqués aussi bien pour les fentes unilatérales que bilatérales. Des bandes élastiques assurant la rétention de l'orthèse peuvent être associées à des boutons extraoraux servant d'ancrage sur les plaques.

Il serait simpliste de penser que le traitement des fentes orofaciales est terminé lors de la dernière intervention de la correction primaire; un suivi interdisciplinaire de l'enfant s'impose pour déceler et traiter les séquelles du traitement primaire et celles plus tardives, inhérentes à la malformation. La prise en charge, par un seul spécialiste, d'un enfant porteur d'une fente orofaciale est une aberration. L'organisation d'une consultation multidisciplinaire qui regroupe tous les spécialistes compétents dans le traitement des pathologies engendrées par la malformation est indispensable.

\section{CONCLUSION}

La prise ne charge prothétique néonatale des fentes orofaciales qu'elles soient syndromiques ou non, influence véritablement la thérapeutique générale de ces défauts en réduisant au maximum les troubles qui leurs sont liés et en favorisant une croissance normale du massif facial. Cette thérapeutique précoce ne constitue qu'une partie du traitement global qui comporte plusieurs intervenants selon une succession chronologique bien définie, notamment le chirurgien, le pédodontiste, l'orthodontiste et l'orthophoniste. L'évaluation de ce traitement ne peut être envisagée donc qu'à l'âge adulte. À cet âge, la réhabilitation prothétique peut être une seconde fois entamée, c'est le cas des fentes séquellaires qui siègent fréquemment au niveau palatin, on parle alors de la prothèse vélopalatine. 


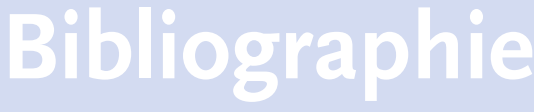

[1] Baylis A. Head and Neck Embryology : An Overview of Development, Growth and Defect in the Human Fetus, 2009.

[2] Buys R, et al. Fentes faciales - La séquence de Pierre Robin : choix parmi plusieurs protocoles de traitement. Archives de Pédiatrie 2010 ; 17(6): :787-788.

[3] Wei T, et al. Association analysis between the IRF6 G820A polymorphism and nonsyndromic Cleft Lip and/or Cleft Palate in a Chinese Population. Cleft Palate-Craniofacial Journal 2009 ; 46 (1): 89-92.

[4] Griti-linde A. The etiopathogenesis of cleft lip and cleft palate : usefulness and caveats of mouse models. Current Topics in Developmental Biology 2008 ; 84:37-138.

[5] Beumer J, et al. Maxillofacial

Rehabilitation : Prosthodontic and Surgical
Management of Cancer-Related, Acquired, and Congenital Defects of the Head and Neck, 2011.

[6] Banu K, et al. A preoperative appliance for a newborn with cleft palate. Cleft palateCraniofacial Journal 2009 ; 46(1): 53-57.

[7] Long RE, et al. The americleft Study : An Inter-Center Study of Treatment Outcomes for Patients with unilateral cleft lip and palate part 1. Principles and study design. Cleft Palate-Craniofacial Journal 2011 ; 48 (3): 239-243.

[8] Hathaway R, et al. The americleft Study : An Inter-Center Study of Treatment Outcomes for Patients with Unilateral Cleft Lip and Palate Part 2. Dental Arch Relationships. Cleft PalateCraniofacial Journal 2011 ; 48 (3): 244-251

[9] Igal S, et al. A feeding obturator for a preterm baby with Pierre Robin sequence. J Prosthet Dent 2005 ; 93:197-200.

[10] Bault J-P, et al. La face fotale normal et pathologique : Aspects échographiques, 2013
[11] Narendra R, et al. Feeding obturator - a presurgical prosthetic aid for infants with cleft lip and palate - clinical report. Annals \& Essences of Dentistry 2013 ; 5 (2): 1

[12] Chandna1 P. et al. Feeding obturator appliance for an infant with cleft lip and palate. $J$ IndSoc Ped Prev Dent 2011 ; 29 (1): 71-73.

[13] Rashel B.et al. Rehabilitation of one day old neonate with cleft lip and palate using palate obturator : a case report. Inter J Clin Ped Dent $2012 ; 5$ (2): 145-147.

[14] Rizwaan AS. et al. Prosthetic rehabilitation of cleft compromised newborns : a review. Journal of Clinical and Diagnostic Research $2010 ;(4): 3632-3638$.

[15] Noirrit E. et al. Plaques palatines chez le nourrisson porteur de fente labiomaxillaire. EMC 2005 ; 22-066-B-55.

[16] Rizwaan AS et al. Prosthetic Rehabilitation of Cleft Compromised Newborns : A Review. Journal of Clinical and Diagnostic Research 2010 ; 3632 (4):3632-3638. 